\title{
A CRITERIA OF IDEALITY OF MECHANICAL CONSTRAINTS IN PRINCIPLE OF COMPATIBILITY
}

\author{
DINH VAN PHONG \\ Hanoi Technology University
}

\section{§. INTRODUCTION}

With the aids of computers the principle of compatibility is convenient and powerful method for studying motion of mechanical system with constraints. The theoretical basis of method is described in [6]. The great advantage of this method is fact that reaction forces are not excluded from motion equations and so they could be directly computed with other dynamical quantities. But on the other hand the complexity of forms of motion equations doesn't allow its easy application in practice. So just algorithms which could be realized on computer make the method be powerful tool. Some examples of this trend were showed in $[4,5]$.

As showed in $[4,5,6]$ a criteria of ideality of constraints is one of key problems. With reaction forces in motion equations and the equations of constraints we have a under determined system of equations. So in order to determine completly the motion of system we must add the auxilary conditions. And in our case these conditions will be the ideality of constraints of the Appell-Frzeborski-Chetaev's type.

In this article we will show the theoretical basis and algorithms for realizing these conditions in system dynamical solution on computers.

\section{§2. CRITERIA OF IDEALITY OF CONSTRAINTS}

Let's consider a mechanical system with $n$ holonomic coordinates

$$
\underline{q}=\left[q_{i}\right], \quad i=1,2, \ldots, n
$$

The constraints of these coordinates could be written in the matrix form:

$$
\underline{B} \cdot \underline{\ddot{q}}+\underline{b}_{0}=0
$$

where

$\underline{B}_{s \times n}$ is matrix of coefficients, its elements are functions of coordinates $q_{i}$ and velocities $\dot{q}_{i}$ $i=1, \ldots, n$

$\underline{b}_{0 s \times n}$ is column vector of coefficients, its elements are functions of $q_{i}$ and $\dot{q}_{i}, i=1, \ldots, n$

$\ddot{q}_{n \times 1}$ is column vector of generalized accelerations

$s$ is number of constraints of system

$n$ is number of holonomic coordinates.

We suppose that the condition (2.1) is well-chosen that means the rank of $B$ is $s$.

Due to these constraints we will have in the motion equations $n$ reactions forces (see [6]):

$$
\underline{R}=\left[R_{i}\right], \quad i=1, \ldots, n \text {. }
$$


According to the Appell-Frzeborski-Chetaev's definition the criteria for ideality of these constraints are:

$$
\delta \underline{q}^{T} \cdot \underline{R}=0
$$

where $\delta q=\left[\delta q_{i}\right], i=1, \ldots, n$ is colum vector of virtual displacements of coordinates.

Because of presence of $s$ constraints conditions (2.1) the system have only $(n-s)$ degrees of freedom. So we should have $(n-s)$ independent pseudoaccelerations which are defined as follows:

$$
\ddot{\Pi}=\underline{E} \cdot \underline{\ddot{q}}
$$

where

$\ddot{\Pi}=\left[\ddot{\Pi}_{i}\right] i=1, \ldots, n-s$ is vector of pseudoacceleration

$\underline{E}_{(n-s) \times n}$ is matrix of well-chosen coefficient.

Then inversely, from (2.1) and (2.3) we can compute generalized accelerations:

$$
\underline{q}=\underline{D} \underline{\Pi}+\underline{d}_{0}
$$

where

$\underline{D}_{n \times(n-s)}$ is matrix of coefficients

$\underline{d}_{0 n \times 1}$ is column vector of coefficients

$\underline{D}$ and $\underline{d}_{0}$ are defined uniquely from (2.1) and (2.3) if $\underline{E}$ and $\underline{B}$ resp. $\underline{b}_{0}$ are already known. Or in other words: matrix $\underline{D}$ and vector $\underline{d}_{0}$ could be chosen arbitrarily with only one condition: for $(n-s)$ independent pseudoaccelerations $\ddot{\Pi}_{i}, i=1, \ldots, n-s$ the accelerations $\ddot{q}_{i} i=1 \ldots, n$, derived from (2.4) must satisfy the constraints (2.1). It's clear that from (2.4) we can write for $\delta \ddot{q}$ :

$$
\delta \ddot{q}=\underline{D} \cdot \delta \ddot{\Pi}
$$

Before showing how we can choose matrix $\underline{D}$ in next paragraph we will proove that condition (2.2) can be replaced by another formulation with presence of $\underline{D}$.

Really, the condition (2.2) could be written in the form (Gauss definition):

$$
\delta \underline{q}^{T} \cdot \underline{R}=0
$$

and with pseudoaccelerations and (2.5) we have:

$$
\delta \underline{\Pi}^{T} \cdot \underline{D}^{T} \cdot \underline{R}=0
$$

Now, because of independence of $\delta \ddot{\Pi}^{T}$ we have a criteria of ideality of constraints in form:

$$
\underline{D}^{T} \cdot \underline{R}=0
$$

where $\underline{D}$ is matrix of dimension $(n-s) \times n$ and $\underline{R}$ of dimension $n \times 1$.

This matrix equation give $(n-s)$ algebraic equations (in general nonlinear) which complet the system of motion equations of mechanical system under consideration. For the automatic generation of equations on computer we should have the matrix $\underline{D}$ uniquely defined. In next paragraph we will consider the algorithm and all problems concerning.

\section{§3. ALGORITHM}

First we will show how the matrix $\underline{D}$ could be chosen, Let's multiply both sides of (2.4) by matrix of constraints coefficient $\underline{B}$. This operation is valid as $\underline{B}$ is of dimension $s \times n$ and $\underline{q}$ resp. $\underline{D} \cdot \underline{\ddot{I}}$ or $\underline{d}_{0}$ of dimension $n \times 1$ :

$$
\underline{B} \underline{\ddot{q}}=\underline{B} \underline{D} \underline{\underline{I}}+\underline{B} \underline{d}_{0}
$$


with regards to $(2.1)$ we get:

$$
-\underline{b}_{0}=\underline{B} \underline{D} \underline{\Pi}+\underline{B} \underline{d}_{0}
$$

If $\underline{D}$ and $\underline{d}_{0}$ were chosen as following:

$$
\underline{B} \underline{d}_{0}=-\underline{b}_{0}
$$

and

$$
\underline{B} \underline{D}=0
$$

then (3.1) should be satisfied.

Matrix equation (3.2) and (3.3) represents two separate systems of algebraic.equations. Both are underdetermined so really we can speak about choix of coefficient $\underline{d}_{0}$ and $\underline{D}$. Let's study equation (3.3) more in detail.

The matrix equation (3.3) presents $s \times(n-s)$ equations for $n \times(n-s)$ unknowns. We can formul the problem in another way: to find $(n-s)$ vectors $\underline{d}_{1}, \ldots, \underline{d}_{n-s}$ of dimension $n$, which should be orthogonal to $s$ vectors $\underline{b}_{i}, i=1, \ldots, s$, of dimension $n$. So we have matrices $\underline{B}=\left[\underline{b}_{i}^{T}\right]$ and $\underline{D}=\left[\underline{d}_{i}\right]$. If we add the conditions of orthogonality of vectors $\underline{d}_{i}, i=1, \ldots, n$ to each other, we will get the task which could be solved consecultively for $i=1, \ldots, n-s$ : define new vector which is orthogonal to previously defined vectors.

Now we have algorithm for defining matrix $\underline{D}$ :

1. Define $s$ vectors $\underline{b}_{i}$ of dimension $n, i=1,2, \ldots, s$

2. Set

$$
\begin{aligned}
m & =s \\
k & =1 \\
\underline{a}_{i} & =\underline{b}_{i} \quad \text { for } \quad i=1,2, \ldots, m
\end{aligned}
$$

3. Find vector $p$ of dimension $n$ from underdetermined (or determined) system of equations:

$$
\underline{a}_{i} \underline{p}=0, \quad i=1,2, \ldots, m
$$

(in other words: find vector $\underline{p}$, orthogonal to vector $\underline{a}_{i}, i=1, \ldots, m$ )

4. Transform vector $p$ in orthonormal vector:

$$
\underline{p}^{T} \cdot \underline{p}=1
$$

5. Set $\underline{d}_{k}=p$

6. Set $m=m+1$

$$
k=k+1
$$

7. If $m>n$ then stop

otherwise set $\underline{a}_{m}=p$ and continue algorithm from step 3 .

Note that $\underline{a}_{i}, p$ are only working vectors.

Affter stopping algorithm we have defined $(n-s)$ vectors $\underline{d}_{k}, k=1, \ldots,(n-s)$. The matrix $\underline{D}$ consists of $(n-s)$ columns $\underline{d}_{k}$. Or for using in the equation $(2.8): \underline{D}^{T}$ is created by $(n-s)$ rows $\underline{d}_{k}^{T}$.

The last discussion is devoted step 3 of our algorithm. The problem of solving determined. or undetermined linear algebraic system of equations presents a special class of numerical mathemathics. In latest years the ABS methods, due to Abaffy. Broyden and Spedicato [1], for finding solution of mentioned systems, are subject of many research works, see e.g. $[2,3]$. In this class of methods we use a special interate algorithm, so-called symetric or Huang algorithm. This algorithm in original finds a minimum Eucledean norm of solution i.e. among all orthogonal vectors which satisfies undetermined system of equations: 
where

$\underline{A}_{m \times n}$ is matrix of coefficients of dimension $m \times n$

$\underline{x}_{n \times 1}$ is column vector of dimension $n \times 1$

$c_{m \times 1}$ is right side vector of dimension $m \times 1$

Direct applying this algorithm doesn't give the expected results: as in our case $\underline{c}=0$, this leads to the trivial solution of (3.4) $\underline{x}=0$, i.e. $\underline{D}=0$ for (2.8), what we don't want. So modification of the algorithm should be done, due some conditions of initial iteration $\underline{x}^{0}$. The realization on computers showed that these modifications are satisfying.

\section{\$4. RESULTS ON COMPUTERS AND CONCLUSION}

The considered algorithm was tested in some applications of mechanical systems. It creates a special module of program which generates automatively equations of motion by means of principle of comptability and then determines all coordinaters and reaction forces.

In general we note that the algorithm enables the generation of motion equation more easy as we can choose coordinates arbitrarily and then add auxilary constraints conditions. Even more in the case of so-called "program constraint" this is nearly only way to solve the problem.

The program with considered algorithm was tested in $286,386,486$ based personal computers and showed good results, see e.g. $[4,5]$.

This publication is completed with financial support from the National Basic Research Program in Natural Sciences.

\section{REFERENCES}

1. Abbaffy J., Broyden C. G., Spedicato E. A class of direct methods for linear systems. numerisch Mathematik, 45 (1984) 3, 361-387.

2. Bodon E. An algorithm for solving determined or undetermined, full or deficient rank linear systems based upon the optimally conditioned ABS algorithm. Optimization, 21 (1990) 2, $237-246$.

3. Bodon E. A code for linear least equares based upon the ABS procedures. Optimization, 21 (1990) 3, 371-378.

4. Do Sanh, Dinh Van Phong. The study of equation of motion and inovating a trolley collector. Proceedings of fifth national conference on mechanics, Hanoi, 1993.

5. Do Sanh, Dinh Van Phong. Dynamical calculation for a vibration sieve of a grinding machine. Proceedings of fifth national conference on mechanics. Hanoi, 1993.

6. Do Sanh. On the motion of constrained mechanical systems. The thesis of doctor of science. Technical University of Hanoi, Hanoi, 1980.

Received October 12, 1999

\section{ĐIỀU KIỆN LÝ TƯởNG CƯA CÁC LIÊN KẾT TRONG NGUYÊN LÝ PHÙ HợP}

Bài báo xây dựng một thuật toán cho phép thiết lập một cách tự động các phương trình suy ra từ điều kiện lý tướng của các liên kết. Trên cơ sớ lý thuyết được trình bày dưới dạng ma trận, thuật toán được viết cho máy tính. Một số vấn đề về phương pháp số liên quan cũng được đề cập đến. 